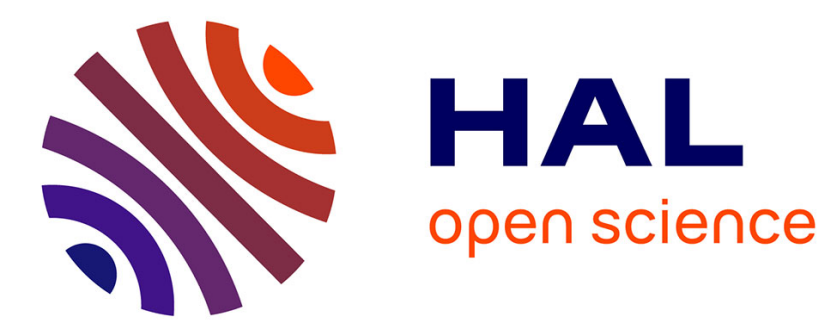

\title{
The self-organization of ball bouncing
}

Guillaume Avrin, Isabelle Siegler, Maria Makarov, Pedro Rodriguez-Ayerbe

\section{To cite this version:}

Guillaume Avrin, Isabelle Siegler, Maria Makarov, Pedro Rodriguez-Ayerbe. The self-organization of ball bouncing. Biological Cybernetics (Modeling), 2018, 10.1007/s00422-018-0776-8 . hal-01872211

\section{HAL Id: hal-01872211 \\ https://hal.science/hal-01872211}

Submitted on 11 Sep 2018

HAL is a multi-disciplinary open access archive for the deposit and dissemination of scientific research documents, whether they are published or not. The documents may come from teaching and research institutions in France or abroad, or from public or private research centers.
L'archive ouverte pluridisciplinaire HAL, est destinée au dépôt et à la diffusion de documents scientifiques de niveau recherche, publiés ou non, émanant des établissements d'enseignement et de recherche français ou étrangers, des laboratoires publics ou privés. 


\title{
The self-organization of ball bouncing
}

\author{
Guillaume Avrin • Isabelle A. Siegler • Maria Makarov • Pedro Rodriguez-Ayerbe
}

Received: date / Accepted: date

\begin{abstract}
The hybrid rhythmic ball-bouncing task considered in this study requires a participant to hit a ball in a virtual environment by moving a paddle in the real environment. It allows for investigation of the on-line visual control of action in humans. Changes in gravity acceleration in the virtual environment affect the ball dynamics and modify the ball-paddle system limit cycle. These changes are shown to be accurately reproduced through simulation by a model integrating continuous information-movement couplings between the ball trajectory and the paddle trajectory, giving rise to a resonance tuning phenomenon. On the contrary, the tested models integrating only intermittent sensorimotor couplings were unable to replicate the observed human behavior. Results suggest that the visual control of action is achieved on-line, in a prospective way. Human rhythmic motor control would benefit from the timing and phase control emerging from the low-level continuous coupling between the central pattern generator and the visual perception of the ball trajectory. This control strategy, which precludes the need for internal clock and explicit environmental representation, is also able to explain the empirical result that the bounces tend to converge toward a passive stability regime during human ball bouncing.
\end{abstract}

This work was supported by the Foundation for Scientific Cooperation (FSC) Paris-Saclay Campus.

Guillaume Avrin, Maria Makarov, Pedro Rodriguez-Ayerbe

Laboratoire des Signaux et Systèmes (L2S), CentraleSupélec- CNRSUniv. Paris-Sud, Université Paris-Saclay, F-91192 Gif-sur-Yvette, France Tel.: +33169851712

E-mail: guillaume.avrin@gmail.com

Guillaume Avrin, Isabelle A. Siegler

CIAMS, Univ. Paris-Sud, Université Paris-Saclay, 91405 Orsay, France

Guillaume Avrin, Isabelle A. Siegler

CIAMS, Université d'Orléans, 45067 Orléans, France
Keywords Ball bouncing · Information-movement couplings $\cdot$ Neural oscillators $\cdot$ Dynamic systems $\cdot$ Resonance tuning

\section{Introduction}

Humans have the capacity to synchronize limb movement with rhythmic visual stimuli. This capacity has been demonstrated for tasks such as rhythmic finger tapping and limb oscillation synchronized with a ball bouncing or flashing lights (Gan et al 2015; Iversen et al 2015). Electrophysiologybased studies have shown that some rhythmic movements are the result of rhythm generators, known as Central Pattern Generators (CPG). CPG dynamics are modulated by sensory signals through low-level information-movement couplings (Pearson 2004) and descending signals from the cerebrum (Grillner 2006; Harris-Warrick 2011; Rossignol et al 2006). A topical issue is to determine if the information-movement couplings involved in the timing control of rhythmic tasks are intermittent or continuous (Van Der Steen and Keller 2013; Torre and Balasubramaniam 2009).

Among on-line visual control approaches, the information processing hypothesis considers that during limb synchronization with external periodic signals, movement characteristics such as period or phase are corrected once per cycle, through sequential relations called intermittent couplings (Van Der Steen and Keller 2013). Most of the models of human behavior during rhythmic impact tasks such as finger tapping (see Repp (2005) for a review) and bouncing (de Rugy et al 2003), consider intermittent couplings between the agent and the environment. These discrete-time couplings, which generally take the form of intermittent parametric control laws, certainly play a role in the achievement of rhythmic tasks (Siegler et al 2013; Warren 2006). They have recently 
been used to model the paddle period adaptation during ball bouncing in Avrin et al (2016, 2017a); de Rugy et al (2003).

However, these intermittent couplings might be complemented by emergent dynamics arising from continuous interactions between the CPG and other oscillators from the Neuro-Musculo-Skeletal system (NMS) or from the environment when sensory information is continuously available. These continuous couplings have been called global entrainments or continuous state control in the literature (Taga 1995; Warren 2006). They give rise to entrainment and resonancetuning phenomena observed during the rhythmic visuomotor tasks performed by humans. Interestingly, the phase- and frequency-locking phenomena resulting from the resonancetuning phenomenon lead the coupled oscillators to converge toward the resonant frequency of the closed-loop system, thus leading to energy-efficient and stable movements (Williamson 1998; Goldfield et al 1993; Rabinovich et al 2006; Russell and Sternad 2001). Resonant-tuning phenomena have particularly been evidenced for couplings involving proprioception during free limb oscillation tasks (Hatsopoulos and Warren Jr 1996; Goldfield et al 1993; Williamson 1998). Resonance tuning and entrainment phenomena were also observed during visuomotor tasks such as locomotion (Pelah et al 2015), postural sway (Bertenthal et al 1997; Dijkstra et al 1994b,a), synchronization of the human arm oscillation with an oscillating external event (Buekers et al 2000; Lopresti-Goodman et al 2008; Schmidt et al 2007; Varlet et al 2014, 2012; Washburn et al 2014; Gan et al 2015; Hove et al 2013; Iversen et al 2015), interpersonal visual coordination of limb oscillations (Oullier et al 2008; Schmidt et al 1998; Schmidt and Turvey 1994; Schmidt et al 1990) and during visuomotor tracking of a sinusoidally moving target (Russell and Sternad 2001; Wimmers et al 1992).

During a one-dimensional (vertical) ball-bouncing task, a participant hits a ball with a paddle to achieve a specific target height (see Fig. 1). This task, which needs precise sensorimotor synchronization to adapt the paddle period to the ball period at each cycle, has often been used to investigate the information-movement couplings involved in the human control of rhythmic hybrid tasks (Ankarali et al 2014; Avrin et al 2017b; Bazile et al 2013, 2016; Marchal-Crespo et al 2015; Morice et al 2007; Ronsse et al 2010; Ronsse and Sternad 2010; Schaal et al 1996; Siegler et al 2010, 2013; Sternad et al 2001; Wei et al 2007, 2008). These couplings have efficiently been modeled by both intermittent and continuous couplings. However, no study has ever clarified the interest of relying on one kind of coupling over the other.

Considering models implementing intermittent couplings, de Rugy et al (2003) proposed a control architecture relying on a Matsuoka oscillator generating almost sinusoidal paddle trajectories with an oscillator frequency adapted intermittently to control the paddle period. An extension of this controller integrating a paddle amplitude adaptation law was proposed in Avrin et al (2016) for torque control and Avrin et al (2017a) for position control. These three models adapted the paddle period directly after impact to equal the predicted ball period based on the perception of the ball velocity after impact. If one of these models were strictly implemented by the nervous system to control the task, then humans would need to have an internal model of the gravity acceleration and of the mathematical relation between the post-impact velocity of the ball and the ball period to predict ball flight duration just after impact and ball launch. The hypothesis that humans predict the ball period based on an explicit internal representation of the gravity acceleration and use this information to achieve the task by relying on once-per-cycle intermittent coupling is tested in this study. In Avrin et al (2017b), the possibility that the paddle period was adapted only when the ball reaches its apex was investigated. In that case, the agent may deduce the ball period as the double of the half-period between impact and ball apex. This strategy would thus preclude the need for prediction and internal representation of the gravity acceleration. It was shown in this previous study that under this control strategy, the bounce was unstable, thus discrediting this hypothesis.

Instead of relying on internal models, it is possible that human control strategies rely on resonance phenomena resulting from continuous couplings between the NMS and the environment. This solution was implemented in Avrin et al (2017b) to synchronize the paddle trajectory with the ball trajectory. It demonstrated an accurate matching with the humans' bounce error series for trials including perturbations on the environmental conditions determined by the gravity acceleration and the ball-paddle restitution coefficient. As a consequence, concerning the paddle period adaptation law, two competing hypotheses exist. The first one is the intermittent parametric control of the paddle period, directly after impact, which assumes knowledge about ballistic flight and gravity acceleration. The second one is the state control of the paddle period relying on the continuous coupling between the ball trajectory and the paddle trajectory, giving rise to a resonance-tuning phenomenon.

Furthermore, it has been shown that during ball bouncing, human behavior is articulated around a passive stability regime evidenced by the stability analysis of the task dynamics (Schaal et al 1996; Sternad et al 2001; Tufillaro et al 1986). Indeed, if the paddle hits the ball in a specific portion of the paddle cycle, then small perturbations die out without requiring any active control of the paddle trajectory. The ball returns to its pre-perturbation limit cycle after a few transient cycles because of the passive dynamics of the task. This passive stability regime corresponds to a specific interval of paddle acceleration at impact, which depends on the ballpaddle restitution coefficient and on the gravity acceleration. Humans were also shown to actively control the paddle trajectory based on sensory information, resulting in bounces 
staying or going back to this passive stability regime (de Rugy et al 2003; Morice et al 2007; Siegler et al 2010; Wei et al 2007). The nature of the information-movement couplings involved in this convergence process remains undetermined. An active control of the paddle acceleration at impact (or impact phase) could be responsible for convergence toward a limit cycle inside the passive stability regime. An alternative hypothesis is that the limit cycle emerges from the continuous interaction between the NMS and the ball. The self-organization of the coupled ball-paddle system would give rise to phase-locking and frequency-locking phenomena. The convergence toward a preferential paddle acceleration at impact would be the result of this resonance tuning.

The objective of the present paper is to identify the control principles and information-movement couplings involved in the stabilization of the human ball-bouncing task. More specifically, the first objective is to determine whether these couplings are only intermittent (parametric control) or whether considering continuous couplings (state control) is necessary to explain the observed adaptation of the paddle period to the ball period. The second objective is to determine if an active control of paddle acceleration at impact, through intermittent couplings, is responsible for the observed convergence toward the passive stability regime during human ball-bouncing trials.

These different coupling hypotheses are translated into testable models of human ball bouncing. The candidate models differ from each other by the information-movement couplings involved in the control of the paddle period and acceleration at impact. After presenting arguments discrediting the first hypothesis, involving an intermittent control of the paddle period but neither active nor emergent control of the paddle acceleration at impact (HYP1), with regards to past experimental results, the paper tests three other hypotheses. The second hypothesis (HYP2) is that both the paddle period and acceleration at impact are intermittently controlled and that the active control of the paddle acceleration at impact is responsible for the convergence toward the passive stability regime. The third hypothesis (HYP3) is that a continuous coupling between the perceived ball trajectory and the NMS is responsible for the adaptation of the paddle period to the ball period. The paddle acceleration at impact is not actively controlled but the convergence toward a specific limit cycle emerges from the continuously coupled ball-NMS system dynamics. The last hypothesis (HYP4) is a compromise between the two previous hypotheses: in addition to the continuous coupling between the ball and the NMS, an intermittent coupling controls the paddle acceleration at impact. Therefore, the convergence of the paddle acceleration at impact toward a specific limit cycle results from both active and emergent control.

It is known that gravity affects the human NMS biologically and physically, which in return changes our gait or limb synchronization, as analyzed for walking, running and jumping for expected and unexpected changes in gravity (Dietz and Duysens 2000; Sylos-Labini et al 2013; White et al 2008). Manipulating gravity during rhythmic motor tasks in interaction with the environment allows for a better understanding of the existing interactions between the central nervous system, the musculoskeletal system, and the environment. More generally, it is useful to shed light on the processes involved in the generation of muscular activity patterns and sensory feedback modulation. The human capacity to tune the steady-state bouncing inside the passive stability regime and the influence of gravity on this limit cycle will be the main elements used to discriminate between the intermittent or continuous coupling hypotheses. This paper proposes to use gravity manipulation to confront the competing hypotheses and to explain the convergence of the bounces toward the passive stability regime.

To summarize, the objective of this paper is to discriminate information-movement coupling models that have been mentioned in the literature about the ball-bouncing task, by implementing these hypotheses into testable models and by comparing their predictions with the experimental data. This confrontation should lead to a deeper understanding of the brain-body-environment interactions as well as open up avenues for the design of bioinspired robot control algorithms. Section 2 presents the candidate CPG-based control architectures and the discrimination method. Section 3 presents the results of comparisons between the model and the experimental data and Section 4 discusses these results. Conclusions are drawn in Section 5.

\section{Methods}

\subsection{Candidate models of human ball bouncing}

The ball-paddle impact law, the CPG model, the arm mechanical system and the amplitude adaptation law presented below are shared by the candidate models.

\subsubsection{The bouncing ball model}

During the ball-bouncing task, the participant oscillates his/her forearm (rotation at the elbow) to bounce a ball vertically so that the ball apex reaches a predefined target height $h_{p}$ at each cycle. The article uses the following notations (Fig. 1): $\theta$ is the angle between the horizontal axis and the forearm, $T_{b}(k)$ is the ball period during cycle $k$, i.e. between impact $k$ and impact $k+1 . T_{r}(k), A C_{r}$ and $\varepsilon(k)$ are the paddle period, the paddle acceleration at impact and the bounce error of cycle $k$, respectively. The bounce error is defined by the distance between the ball apex $h_{a}(k)$ and the target height $h_{p}$, i.e. $\varepsilon(k)=h_{a}(k)-h_{p}$. 
The ball flight between impacts is governed by ballistic equations:

$$
\left.\begin{array}{l}
X_{b}(t)=X_{b}(k)+V_{b}(k) t-0.5 g t^{2} \\
V_{b}(t)=V_{b}(k)-g t
\end{array}\right\} \text { for } t_{k}<t<t_{k+1},
$$

in which $g$ denotes the gravity acceleration, $X_{b}(t)$ the ball position, $t_{k}$ the $k$-th impact instant, $X_{b}(k)$ the $k$-th impact position and $V_{b}(k)$ the ball velocity directly after impact $k$. The impact equation is:

$V_{b}(k)=-\alpha V_{b}(k)^{-}+(1+\alpha) V_{r}(k)$,

in which $\alpha$ is the ball-paddle restitution coefficient at impact, $V_{r}(k)$ the paddle velocity at impact and $V_{b}(k)^{-}$the ball velocity directly before impact $k$.

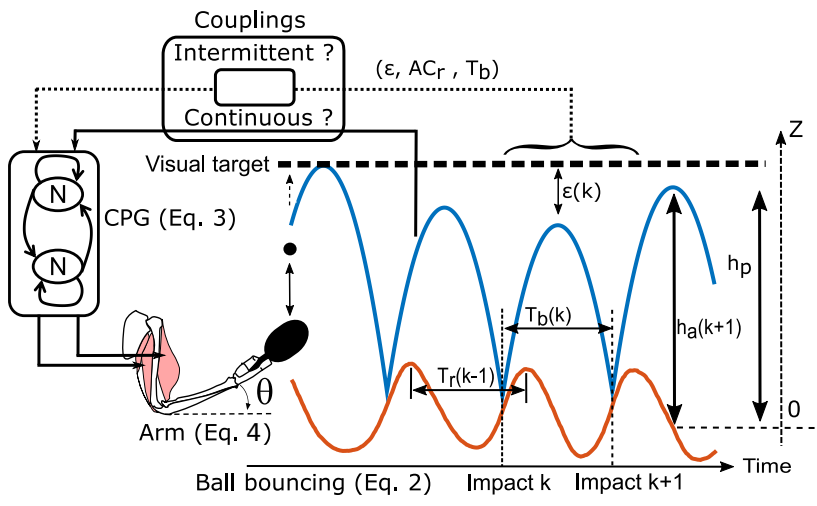

Fig. 1: Model of the human control architecture during ball bouncing (see Section 2.1.1 for legends).

\subsubsection{The neuromechanical model}

The neuromechanical model (CPG and arm) shared by the various models tested in this study is similar to the model presented in Avrin et al (2017b). A Matsuoka neural oscillator acts as a CPG and activates the arm flexor and extensor muscles to generate oscillating torque at the elbow (Matsuoka 1985, 2011). This oscillator constitutes a parsimonious halfcenter structure generating movements, with profiles encoded on the CPG attractor dynamics, that can be scaled based on information from the target (i.e. bounce error). This CPG model has already been attested to model rhythmic movement generation during different tasks including ball bouncing (de Rugy et al 2003; Taga 1995; Zhang et al 2009). The two neurons in reciprocal inhibition of the Matsuoka oscillator are defined by nonlinear differential equations:

$$
\begin{aligned}
& \tau_{r} \dot{x}_{1}=-x_{1}-\beta v_{1}-\rho y_{2}-h_{0}[m]^{+}+u, \\
& \tau_{a} \dot{v}_{1}=-v_{1}+y_{1}, \\
& \tau_{r} \dot{x}_{2}=-x_{2}-\beta v_{2}-\rho y_{1}-h_{0}[m]^{-}+u, \\
& \tau_{a} \dot{v}_{2}=-v_{2}+y_{2},
\end{aligned}
$$

in which $x_{i}(t)$ and $v_{i}(t)$ are the $\mathrm{i}$-th neuron membrane potential and the self-inhibition responsible for the fatigue phenomenon, respectively. The coupling between neurons is ensured by the terms $y_{i}(t)=\max \left(x_{i}(t), 0\right)$. CPG output is $y_{\text {out }}(t)=$ $\max \left(x_{1}(t), 0\right)-\max \left(x_{2}(t), 0\right)$ and $m(t)$ is CPG sensory input with $[m(t)]^{+}=\max (m(t), 0),[m(t)]^{-}=\max (-m(t), 0) . \rho$ is the mutual-inhibition intensity and $\beta$ the self-inhibition intensity. $u$ is the excitability determining oscillator output amplitude and $h_{0}$ is a constant gain on the input $m(t) . \tau_{r}$ and $\tau_{a}$ are the time constants determining the responsiveness of $x_{i}$ and $v_{i}$, respectively.

The Matsuoka oscillator has two operating modes. In the first mode, the oscillator autonomously produces a periodic limit cycle with a natural pulsation denoted $\omega_{n}$ in the absence of rhythmic sensory input $(m=0)$. In the second mode, the oscillator can be entrained by an external signal or dynamic system to which it is coupled by the input $m$ in a robust and stable way.

Concerning the forearm mechanical impedance, it is a simplified model, linearized around the resting position $\theta=0$, with constant coefficients:

$I \ddot{\theta}+\gamma \dot{\theta}+K \theta=h_{1} \zeta$,

in which $\zeta$ denotes the elbow torque, $I$ the arm inertia, $\gamma$ the damping ratio, $K$ the arm stiffness and $h_{1}$ a constant multiplicative gain on the torque input. The parameter values are taken equal to the values used in Avrin et al (2017b): $K=25 \mathrm{~kg} \cdot \mathrm{m}^{2} \cdot \mathrm{s}^{-2}, \gamma=1.8 \mathrm{~kg} \cdot \mathrm{m}^{2} \cdot \mathrm{s}^{-1}, I=0.1 \mathrm{~kg} \cdot \mathrm{m}^{2}$.

\subsubsection{The paddle amplitude and period control}

Siegler et al (2013) revealed that the control of the paddle trajectory by participants is achieved visually, on a cycleby-cycle basis. During each cycle, the period of the paddle oscillation $T_{r}$ is modulated to match the period of the ball $T_{b}$ and the paddle velocity from previous impact is adapted proportionally to the bounce error $\varepsilon$. The equations summarizing these results are:

$T_{r}(k+1)=T_{b}(k+1)$,

$\Delta V_{r}(k+1) \widehat{=} V_{r}(k+1)-V_{r}(k)=\Lambda_{v e l} \varepsilon(k)$,

in which $\Lambda_{v e l}$ is a negative constant and $V_{r}(k)$ the paddle velocity at impact $k$. In the virtual environment, the paddle trajectory is unaffected by the impact with the ball. As a consequence, the paddle velocity just before impact is equal to the paddle velocity just after impact. The different hypotheses concerning the control paradigms giving rise to these information-movement couplings are presented below.

Error-to-target correction law derived from past experimental results The error-to-target related information-movement coupling of Equations 5 is implemented via an adaptation of the joint torque magnitude at the elbow (i.e. the magnitude 
of the oscillator output), which is determined by the neural oscillator excitability $u$. Once per cycle, when the bounce error is perceived (i.e. when the ball reaches its apex), $u$ is adapted according to the relation $u(k+1)=\lambda \varepsilon(k)+u(k)$. This error-to-target correction law is common to all versions of the model proposed in this paper.

Control of the ball-paddle impact timing Different operating modes are considered for the Matsuoka oscillator to achieve the ball-paddle impact timing control. First, the Autonomous-Oscillation mode (AO) is considered (see details about the autonomous Matsuoka oscillator tuning in Avrin et al (2016)). The information-movement coupling responsible for the paddle period adaptation, presented in Equations 5, is implemented in this operating mode via an adaptation of the oscillator natural frequency. As demonstrated in Avrin et al $(2016,2017 \mathrm{~b})$, this operating mode is robust only if the oscillator natural frequency is adapted directly after impact. This post-impact adaptation requires the ball period to be predicted based on the perception of the ball's post-impact velocity and the internal quantitative knowledge of the gravity acceleration value, as hypothesized in de Rugy et al (2003). This adaptation is achieved by a modification of the oscillator time constants $\tau_{r}$ and $\tau_{a}$ as explained in Avrin et al (2016) and recalled in Equations 6. This mode is used in HYP1 and HYP2.

The alternative operative mode is the Forced-Oscillation mode (FO), where the oscillator is coupled to the perception of the ball trajectory (for instance the ball position or velocity). The paddle period adaptation would thus result from the oscillator entrainment by this informational input. However, this mode is only efficient when the oscillator natural frequency $\omega_{n}$ is close to the ball frequency $2 \pi / T_{b}$ as explained in Avrin et al (2017b). As a consequence, the FO mode is completed by an adaptation of the oscillator natural frequency to equal the ball frequency at each cycle. The oscillator natural frequency adaptation is achieved when, or after, the ball reaches its apex. This FO mode with oscillator natural frequency adaptation is referred to as Mixed-Oscillation mode (MO). This mode is used in HYP3 and HYP4.

For the MO mode implemented in this paper, the continuous oscillator input $m$ is equal to the perceived ball velocity delayed by a duration $t_{d}: m(t)=V_{b}\left(t-t_{d}\right) . t_{d}$ will be referred to as visual time delay in this paper. This delay affects the ball-paddle impact phase as presented in Avrin et al (2017b). To summarize, the oscillator dynamics are modulated by sensory information via Equations 6.

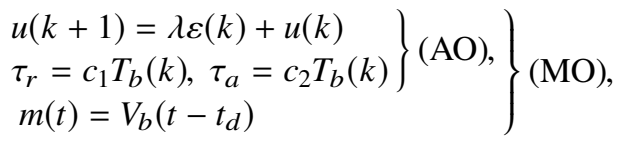

in which $c_{1}$ and $c_{2}$ are two constants parameters chosen so that the natural frequency of the oscillator is equal to $2 \pi / T_{b}$. The tuning method is presented in Avrin et al (2016).

\subsubsection{Active control of the paddle acceleration at impact}

The AO and MO modes can be completed by an additional information-movement coupling responsible for the control of the paddle acceleration at impact (as in HYP2 and HYP4). The real and desired paddle acceleration at impact are noted $A C_{r}$ and $A C_{r}^{*}$. For the $\mathrm{AO}$ mode, the paddle acceleration at impact can be controlled through a modification of the paddle period adaptation law. Indeed, if $T_{r}$ is adapted to be lower than $T_{b}$ for one cycle, then the bounce will occur later in the paddle cycle and the paddle acceleration at impact will be decreased. Inversely, if $T_{r}$ increases to be higher than $T_{b}$, the paddle acceleration at impact will be increased. The period-related equality in Equation 5 becomes:

$T_{r}=T_{b}+v_{1}\left(A C_{r}-A C_{r}^{*}\right)$.

For the MO mode, this adaptation law cannot be used as the paddle period is determined by the low-level coupling with the ball trajectory, and not by its natural frequency. The paddle acceleration at impact is thus controlled through an adaptation of the visual delay parameter $t_{d}$ that determines the phase shift between the ball trajectory and the paddle trajectory:

$t_{d}(k+1)=t_{d}(k)+v_{2}\left(A C_{r}-A C_{r}^{*}\right)$.

For the simulations implemented in this study, the visual input delay $t_{d}$ of the MO mode has to take values proportional to the sampling period of the simulator $\left(t_{\text {samp }}=0.003 \mathrm{~s}\right)$. As a consequence, the active control of the paddle acceleration at impact in the MO mode is expressed as:

$t_{d}(k+1)=t_{d}(k)+f \operatorname{floor}\left(v_{2}\left(A C_{r}-A C_{r}^{*}\right)\right) t_{\text {samp }}$.

The floor function is the function that takes as input a real number $x$ and gives as output the greatest integer that is less than or equal to $x$. It acts as a threshold in Equation 9. Indeed, a small $v_{2}$ value would lead to a large interval of $A C_{r}$ for which the control of the paddle acceleration at impact is nonactive, whereas a large value would result in an almost always active control. It is thus possible to tune $v_{2}$ to match the participants' bounce error or paddle acceleration at impact series during ball bouncing trials. The tuning method is presented in Section 2.3.

\subsubsection{Emergent control of the paddle acceleration at impact}

For robotic applications, Williamson (1999); Arsenio (2000) suggested using the phenomenon of frequency locking occurring when the Matsuoka oscillator is coupled with a sinus signal with a frequency and amplitude equal to those of the ball. They demonstrated that the limit cycle to which the bounce converges is independent of the trial initial conditions and can be predicted based on a Describing Function Analysis (DFA) of the closed-loop system. In Avrin et al 
(2017a), the authors demonstrated that this frequency-locking phenomenon could also be produced by directly feeding the neural oscillator with the non-sinusoidal ball trajectory (as is the case for HYP3). The independence of the limit cycle from the initial conditions remains as shown in Fig. 2 for various initial ball positions $X 0$. On the contrary, the model implementing HYP1 defines a continuum of limit cycles, as demonstrated in Avrin et al (2017a), and the convergence toward a specific limit cycle depends on the initial conditions of the ball-bouncing trial, as illustrated in Fig. 2.

In addition, using the method proposed in Williamson (1999) and the describing functions of the ball bouncing task given in Matsuoka (2013), the influence of gravity acceleration on the bounce limit cycle generated by the model implementing HYP3 can be evidenced. Fig. 3 compares the predicted and simulated steady-state ball-paddle phase shift and paddle frequency as a function of $g$. The prediction of the steady-state paddle frequency is very accurate whereas a small discrepancy remains between the predicted and simulated ball-paddle phase shift (15\% error).

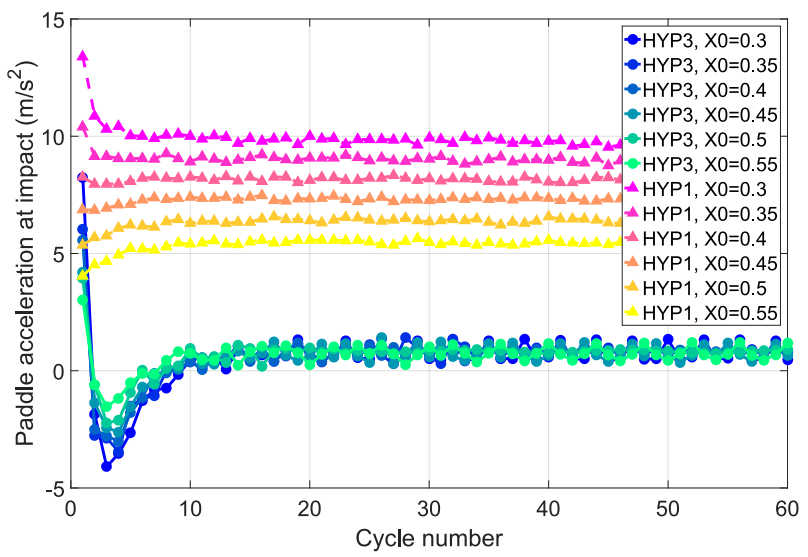

Fig. 2: Influence of the initial ball position $X_{0}$ on the steadystate paddle acceleration at impact, for the models implementing HYP1 and HYP3.

\subsection{Models discrimination method}

\subsubsection{Experimental data used for hypotheses testing}

The models corresponding to the different hypotheses tested in this study are summarized in Fig. 4. To discriminate between these models, this paper analyzes the influence of gravity on the ball-bouncing limit cycle (quantified by the impact phase, position and paddle acceleration) predicted by the different models and observed during the participants' experimental trials. Two types of gravity-related conditions tested in previous experimental papers are used for models
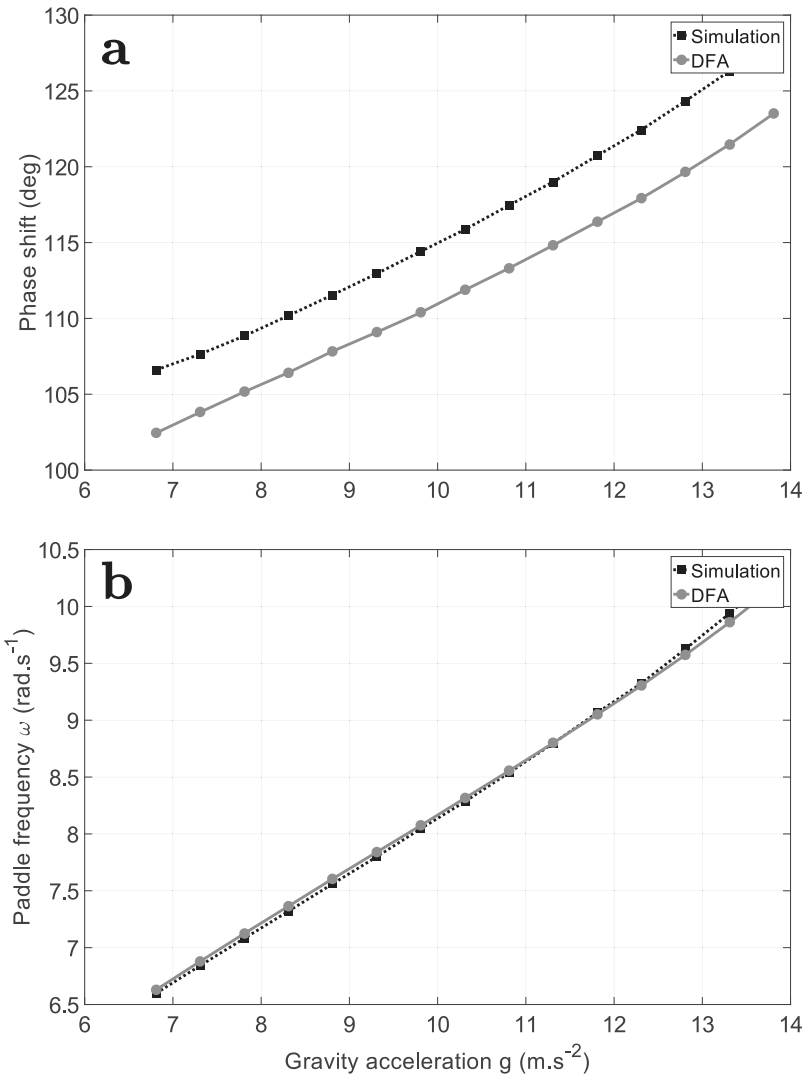

Fig. 3: Comparison of the predicted and simulated steadystate. a: ball-paddle phase shift and b: paddle frequency, as a function of $g$, for the model implementing HYP3.

tuning and discrimination: i) gravity acceleration is changed permanently during on-going trials and ii) it is changed for a one ball cycle during on-going trials and then reset to its preperturbation value. The previous experiments implementing these conditions are described below.

First, during Experiment 2 of Siegler et al (2010), $g$ was suddenly changed during ongoing bouncing and the target height $h_{p}$ was constant and equal to $0.55 \mathrm{~m} .8$ transitions on $g$ were tested. The experiment was completed by 13 participants, each participant performing 12 trials with three perturbations on $g$ separated by 12 or 16 seconds. $g$ was changed as the ball reached its apex. This experiment will be referred to as the " $g$-transition experiment" in this manuscript.

Secondly, to investigate the human bounce error correction strategy after a perturbation, Experiment 2 of Siegler et al (2013) introduced occasional perturbations on $g$ and on the ball launch velocity $V_{b}$ during $40 s$-long trials with a constant target height $h_{p}$ equal to $0.65 \mathrm{~m}$ and environmental conditions before perturbation $g=9.81 \mathrm{~m} . \mathrm{s}^{-2}$ and $\alpha=0.42$. During these trials, the perturbation magnitude $x$ was selected randomly inside the interval $[-0.25,0.25]$ so that, for one full cycle, the ball velocity after impact and the gravity were 
varied to be equal to $V_{b}(k)(1+x)$ and $g(1+x)$, respectively. As a result, the perturbed ball apex $h_{a}^{\prime}$ of the perturbed cycle varied within $\pm 25 \%$ of its original value and the ball period was kept constant. The group of 16 participants was submitted to a total of 4502 perturbations. For results analysis, these perturbations were uniformly binned into ten perturbation magnitude categories (Mag-5, Mag-4, ..., Mag5). This experiment will be referred to as the " $g$-occasional-perturbation experiment" in this manuscript.

Here we assume that humans do not dispose of a learned look-up table where each value of $g$ corresponds to a specific acceleration at impact to be reached. This assumption is supported by the fact that humans are not used to performing rhythmic impact tasks in a variable gravity environment, which would have allowed for the creation of such a table. In addition, during experiments carried out by Siegler et al $(2010,2013)$ the trials were proposed in a random order to participants, thus avoiding any learning or anticipation effect. Based on the experimental results, Siegler et al (2010) also concluded that humans are very unlikely to use an estimated value of the perturbed gravity acceleration to control the paddle movement during on-going ball bouncing. As a consequence, this study does not consider any model integrating such a table.

\subsubsection{Prediction of the different models}

As the paddle acceleration at impact is assumed to be actively controlled in HYP2 and HYP4 to converge toward a specific value, these hypotheses predict that a participant's paddle acceleration at impact will go back to its pre-perturbation value after permanent changes on the gravity during the $g$-transition experiment.

Concerning the ball bouncing task controlled by the model implementing HYP3, the steady-state frequency and phase at impact are shown in Fig. 3 to depend on the gravity value. Thus, the paddle acceleration at impact, which directly depends on the paddle frequency and impact phase for the quasi-sinusoidal paddle movement, also depends on $g$. As a consequence, according to this hypothesis, a transition between the pre-perturbation paddle acceleration at impact and the post-perturbation one is predicted to occur when looking at the human data from the $g$-transition experiment. On the contrary, after the perturbations occurring in the $g$ occasional-perturbation experiment, HYP2, 3 and 4 predict that the post-perturbation paddle acceleration value will be equal to the pre-perturbation value.

HYP1, which considers that the paddle acceleration at impact is not controlled, predicts that after both occasional and permanent perturbation on $g$, the steady-state paddle acceleration at impact will be different from the pre-perturbation value. Indeed, as shown in Fig. 2, convergence toward a specific limit cycle depends on the initial conditions of the ball-bouncing trial for HYP1. It is thus very sensitive to perturbations, either occasional or permanent.

\subsubsection{Statistical tests used for hypotheses testing}

As presented in Section 2.2.2, the tested models predict different influences of the occasional perturbation and permanent transition of $g$ on the pre- and post-perturbation paddle acceleration at impact. The prediction output is binary: either the modification of $g$ modifies the steady-state paddle acceleration at impact, or not. Similarly, for the participants' experimental trials, the pre- and post-perturbation steady-state paddle acceleration at impact will be compared by means of a paired sample t-test. This statistical test will make it possible to evidence whether or not the modification of $g$ significantly modifies the human ball bouncing limit cycle. By comparing model predictions to t-test results, it will be possible to identify which model reproduces the influence of $g$ on the steady-state paddle acceleration at impact.

For $g$-transition experiments, the paired sample t-tests will compare the mean of the 13 participants' means of the first 8 points of the 32 paddle acceleration series to the mean of the 13 participants' means of the last 8 points $(\mathrm{N}=13, \mathrm{p}<0.05)$. During $g$-occasional-perturbation experimental trials, the perturbations were approximately separated by only 8 cycles. As a consequence, the paired sample t-tests will compare the mean of the 16 participants' first point of the 8 paddle acceleration series to the mean of the 16 participants' last point $(\mathrm{N}=16, \mathrm{p}<0.05)$.

\subsection{Tuning of the tested models}

The $g$-transition experiment and the $g$-occasional-perturbation experiment were performed by two different groups of participants. The CPG parameters $c_{1}, c_{2}, \beta, \rho$ are supposed to be independent of the group of participants considered. They are taken equal to the values already used in Avrin et al (2017b) and listed in Table 1. On the contrary, the sensorimotor gains $\left(\lambda, h_{0}, h_{1}, t_{d}\right)$ are assumed to be influenced by participants' expertise in ball bouncing. These parameters were optimized in Avrin et al (2017b) to match the participants' bounce error time series of the $g$-transition experiment Siegler et al (2010). They are thus taken equal to the values found in this previous study. The same parameter setting method is used in this study to match the participants' bounce error time series of $g$-occasional-perturbation experiment Siegler et al (2013). In this tuning method, the parameters $\left\{t_{d}, h_{0}, h_{1}\right\}$ are first optimized by a Particle Swarm Optimization (PSO) (Yagoubi and Sandou 2011) that minimizes the sum of the Normalized Root Mean Square Error (NRMSE) between the steady state time series of the participants and the model position, phase and paddle acceleration at impact. It is important to note that with this optimization, if the bouncing steady-state is 


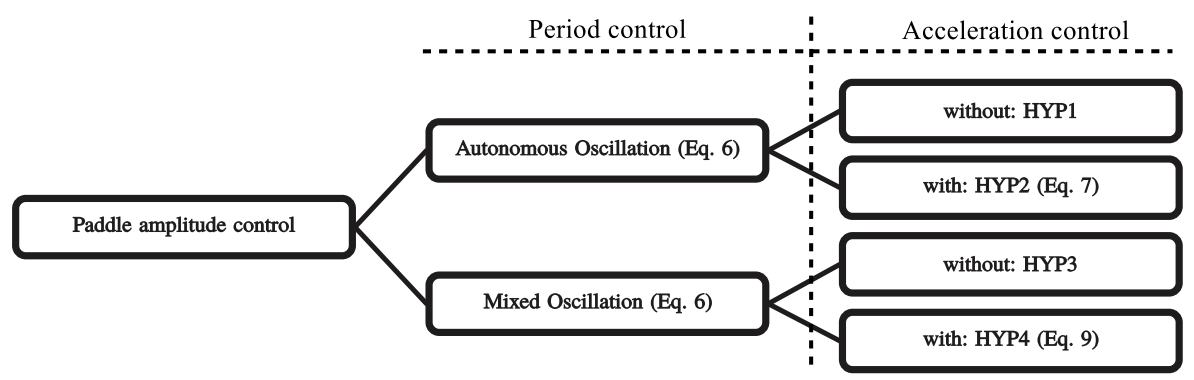

Fig. 4: Models corresponding to the different hypotheses tested.

reached before perturbation, the cost function only evaluates the goodness of the parameters $\left\{t_{d}, h_{0}, h_{1}\right\}$.

This cost function value is independent of parameters $\lambda$, $v_{1}$ and $v_{2}$, which influence the bounce transient state and therefore the response time after perturbations. Once the parameters $\left\{t_{d}, h_{0}, h_{1}\right\}$ were tuned, they were kept constant and the value of $\lambda$ minimizing the sum of the NRMSE between the participants and model bounce errors for the eight cycles around perturbation ( 2 cycles before perturbation and 6 cycles after perturbation) was chosen for tuning. In comparison with the model proposed in Avrin et al (2017b), this paper also considers an intermittent control of the paddle acceleration at impact through the adaptation parameters $v_{1}$ and $v_{2}$. These parameters were optimized to match the participants' behavior observed during the $g$-transition experiment and the $g$-occasional-perturbation experiment. The sum of the NRMSE between the participants and model bounce errors and paddle acceleration at impact for a definite number of cycles around perturbation (32 and 8 cycles for the $g$-transition experiment and $g$-occasional-perturbation experiment, respectively) was calculated for different values of $v_{1}$ or $v_{2}$, depending on the model considered. The $v_{i}$ value leading to the minimum NRMSE was chosen for tuning. Note that the cycles before perturbation will have no influence on the NRMSE if the considered $\lambda, v_{1}$ and $v_{2}$ values allowed the system to reach steady-state before perturbation. Their optimized values are given in Table 1.

For models implementing HYP2 and HYP4, we suppose that the target paddle acceleration at impact $A C_{r}^{*}$ has been learned based on trials with the normal gravity acceleration on Earth $g=9.81 \mathrm{~m} . \mathrm{s}^{-2}$. This gravity condition is the most recurrent in the human behavior experiments used for model discrimination in this study. For this gravity acceleration, in the $g$-transition experiment, the mean paddle acceleration at impact was equal to $-2.71 \pm 1.83 \mathrm{~m} . \mathrm{s}^{-2} . A C_{r}^{*}$ is therefore considered equal to $-2.71 \mathrm{~m} . \mathrm{s}^{-2}$. This value is also close to the maximally stable acceleration at impact $\left(-3 m . s^{-2}\right)$ found with the Lyapunov stability analysis of Sternad et al (2001).
Table 1: Optimized values of the model parameters for the g-transition experiment and g-occasional-perturbation experiment considered in this study

\begin{tabular}{ll}
\hline$g$-transition experiment & $g$-occasional-perturbation experiment \\
\hline$c_{1}=0.137$ & $c_{1}=0.137$ \\
$c_{2}=0.314$ & $c_{2}=0.314$ \\
$\rho=1.689$ & $\rho=1.689$ \\
$\beta=2.512$ & $\beta=2.512$ \\
$h_{0}=96.54$ & $h_{0}=74.98$ \\
$h_{1}=0.610$ & $h_{1}=0.427$ \\
$\lambda=-3.400$ & $\lambda=-10.00$ \\
$t_{d}=36.00 m s$ (for HYP 3 and 4) & $t_{d}=84.00 m s$ (for HYP 3 and 4) \\
$v_{1}=0.004$ (for HYP 2) & $v_{1}=0.005$ (for HYP 2) \\
$v_{2}=0.85$ (for HYP 4) & $v_{2}=0.75$ (for HYP 4) \\
\hline
\end{tabular}

\section{Results}

Table 2 indicates the p-values of the paired sample t-test comparison between the pre-perturbation values and postperturbation values of the paddle acceleration, position and phase at impact, with stars indicating significant differences $(p<0.05)$. In the case of occasional perturbations on $g$, there is no significant difference between the pre- and post-perturbation limit cycles (except for the perturbation conditions $\mathrm{Mag}$-4). Since these perturbations are occasional, they can be seen as a change in the initial conditions of a new trial, which depend on the perturbation magnitude. Thus, these results show that the human limit-cycle is independent of such initial conditions. As a consequence, HYP1, which was shown to depend on initial conditions, is invalidated. From these results, it is certain that the paddle acceleration at impact is controlled, either actively or emergently. This result is in agreement with HYP2, 3 and 4. Simulated time series of the models implementing these three hypotheses and facing the perturbation magnitudes $M a g-5$ and Mag5 of the $g$-occasional-perturbation experiment, are compared to the humans' time series on the top row of Fig. 5 for illustrative purposes. The black solid lines and the shaded region of Fig. 5 indicate the participants' mean paddle acceleration at impact series and standard deviation, respectively. As expected, it can be seen that the post-perturbation paddle 
acceleration at impact of the three models converges toward the pre-perturbation value.

Concerning the $g$-transition experiment, Table 2 indicates that all but one perturbation magnitudes led to a significant difference between the pre- and post-perturbation impact phase. This result suggests that the impact phase was not actively controlled to reach a specific predefined value. It is the main reason why an active paddle acceleration control was considered instead of active phase control in this study. Moreover, among the perturbation conditions tested, 5 out of 8 led to a significant difference between the pre- and post-perturbation paddle acceleration at impact, and 4 out of 8 led to a significant difference between the pre- and postperturbation position at impact, thus making it very unlikely that these variables are actively controlled by humans. These results confirm that the bouncing limit cycle is influenced by the value of the gravity acceleration. They support the hypothesis that humans do not rely on an active paddle acceleration (or phase, or position) control during the ball bouncing task to converge toward a specific limit cycle inside the passive stability regime (invalidating HYP2 and 4). On the contrary, the mixed-oscillation model of HYP3 qualitatively reproduced the influence of $g$ on the steady-state paddle acceleration at impact, without requiring an active control to converge toward a specific steady state.

The paddle acceleration at impact series of the models implementing HYP2, 3 and 4 are compared to the participants' series for the permanent perturbation $13.69 \rightarrow 9.81$ and $9.81 \rightarrow 13.69$ of Experiment 2 of Siegler et al (2010) on the bottom row of Fig. 5 for illustrative purposes. If each model was able to cancel the bounce error after a permanent perturbation on $g$, the model implementing HYP3, which involves the MO mode without active control of the paddle acceleration at impact, better matches the participants' series.

\section{Discussion}

When a perceptual-motor task is weakly constrained by physical and biomechanical systems, the task is generally neutrally stable, meaning that there is no unique fixed point determined by the interaction between the NMS and the environment but, on the contrary, an infinity of equilibrium points. The convergence toward a specific limit cycle thus depends on the initial conditions (Warren 2006). Concerning the ball-bouncing task, it was shown in Avrin et al (2017a), and further demonstrated in this study, that for the human neuromusculoskeletal model considered and under intermittent control of the paddle amplitude and period, the resulting ball-paddle system is neutrally stable. However, during experimental ball-bouncing trials, participants are shown to have preferred limit cycles, as they tend to hit the ball inside the passive stability regime of the ball-bouncing task, independently of the trial initial conditions (Schaal et al 1996; Sternad et al 2001). Thus, human
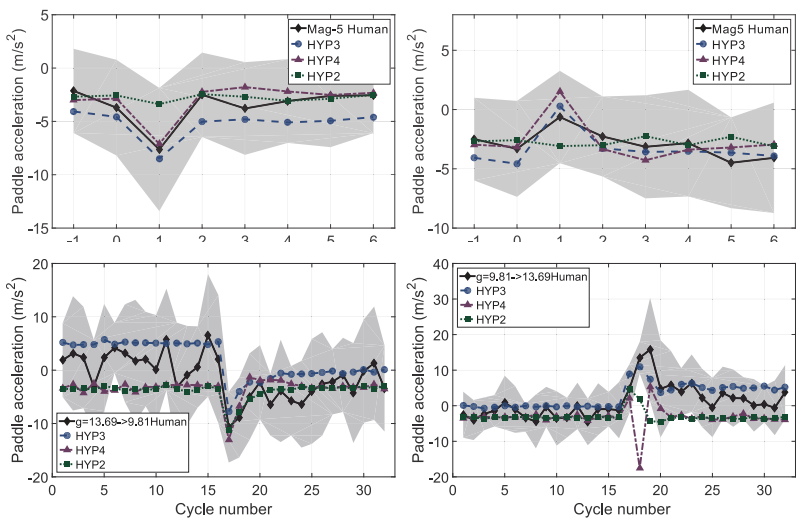

Fig. 5: Comparison of the participants' and model paddle acceleration at impact series after perturbations on $g$. Figures in the top row and bottom row corresponds to occasional perturbations on $g$ and permanent perturbation on $g$, respectively.

ball bouncing is not a neutrally stable task and it is likely that a control strategy is responsible for the convergence toward a specific limit cycle. As this strategy had not previously been identified, this study investigated the different hypotheses concerning its nature. By testing previously proposed models of human ball bouncing, implementing intermittent information-movement couplings (Avrin et al 2016, 2017a; de Rugy et al 2003) and continuous couplings (Avrin et al 2017b), the study makes a contribution to various related ongoing debates about human motor control, as presented below.

\section{Behavioral dynamics rather than information processing}

The present study intends to contribute to the debate opposing the information processing to the behavioral dynamics approaches of the on-line visual control of rhythmic tasks, as presented in the Introduction. Among the models tested in this study, only the model implementing a continuous coupling between the ball and the paddle trajectory, leading to an emergent control of the bouncing limit cycle thanks to phase and frequency locking phenomena, was able to reproduce the observed human behavior. Specifically, it was the only one to explain the influence of the gravity acceleration on the steadystate impact phase, position and paddle acceleration. Thus, these results support the continuous state control hypothesis of the behavioral dynamics theory.

Prospective rather than predictive control An on-going debate exists about the nature of information allowing humans to intercept objects and the way this information is used by the central nervous system. Two main theories compete with each other. The predictive control approach suggests that the brain is able to predict the trajectory of a flying object and 
Table 2: $\mathrm{p}$-values of the paired sample Student t-test comparisons. Stars indicate significant differences $(p<0.05)$.

\begin{tabular}{|c|c|c|c|c|c|c|c|c|}
\hline & \multicolumn{8}{|c|}{$g$-occasional-perturbation experiment $(\mathrm{N}=16)$} \\
\hline & Mag-5 & Mag-4 & Mag-3 & $M a g-2$ & Mag5 & Mag4 & Mag3 & Mag2 \\
\hline$\ddot{X}_{r}$ & 0.56 & $0.04^{*}$ & 0.33 & 0.69 & 0.52 & 0.12 & 0.3 & 0.09 \\
\hline$X_{r}$ & 0.60 & $0.02^{*}$ & 0.18 & 0.74 & 0.77 & 0.59 & 0.40 & 0.13 \\
\hline$\phi$ & 0.64 & $0.02^{*}$ & 0.34 & 0.77 & 0.31 & 0.53 & 0.89 & 0.36 \\
\hline & \multicolumn{8}{|c|}{$g$-transition experiment $(\mathrm{N}=13)$} \\
\hline perturb. $g$ & $9.81 \rightarrow 6.56$ & $9.81 \rightarrow 8.10$ & $11.66 \rightarrow 9.81$ & $13.69 \rightarrow 9.81$ & $9.81 \rightarrow 11.66$ & $9.81 \rightarrow 13.69$ & $6.56 \rightarrow 9.81$ & $8.10 \rightarrow 9.81$ \\
\hline$\ddot{X}_{r}$ & 0.70 & $0.003^{*}$ & 0.15 & $<10^{-3 *}$ & $0.02^{*}$ & $0.01^{*}$ & 0.17 & $0.01^{*}$ \\
\hline$X_{r}$ & 0.23 & 0.35 & $0.003^{*}$ & $0.009^{*}$ & $0.04^{*}$ & $<10^{-3 *}$ & 0.45 & 0.06 \\
\hline$\phi$ & $0.005^{*}$ & $<10^{-3 *}$ & $<10^{-3 *}$ & $<10^{-3 *}$ & $0.001^{*}$ & $<10^{-3 *}$ & $0.003^{*}$ & $<10^{-3 *}$ \\
\hline
\end{tabular}

the appropriate interception position (velocity in the case of hitting tasks) based on internal representations of this object and the environment (Wolpert and Ghahramani 2000). On the contrary, the prospective approach assumes that a continuous coupling exists between the agent and the object to be intercepted. Several interception tasks studying the human capacity to hit (Katsumata and Russell 2012; Lee et al 1983) or catch a ball (Montagne et al 1999; Dessing et al 2005) demonstrated that the human arm movement was guided by the continuous perception of the ball, allowing for such prospective control. Similarly, the ball-bouncing task can be viewed as a rhythmic interception task. After comparing different adaptation strategies, this paper suggests that the paddle period is controlled on-line by the continuous low-level coupling that exists between the ball velocity and the paddle trajectory. The use of such continuous visual information to drive the movement precludes the need for predictive control and thus an internal model of the environment. The CPG entrainment by an input signal takes the form of a reactive control strategy in the sense that there is a tight coupling between the sensory input and the effector output. However, the derivative of a variable indicating the rate of change of this variable, and the input signal being the ball velocity, the CPG benefits from the advantages of both reactive and prospective control strategies. This innovative modeling of the human motor control of rhythmic movement led to results that further support the prospective control hypothesis and more generally the direct perception or ecological approach (Gibson 1979).

The validity of the continuous coupling hypothesis is further supported by the observation that after large perturbations, participants do not wait for the ball to reach its apex to adapt the paddle period to equal the ball period (Avrin et al 2017b). Contrary to what was assumed in previous modeling studies relying on predictive control (Avrin et al 2017a, 2016; Ronsse et al 2010; Ronsse and Sternad 2010; de Rugy et al 2003), this means that humans integrate information from the environment more frequently than just once per cycle. The human on-going movement adaptation during visuomotor tasks was also underlined in Bootsma and Van Wieringen (1990); Zhao and Warren (2015).
Furthermore, a predictive control strategy seems to be less likely to be used by humans during the ball-bouncing task. Indeed, in the $g$-transition experiment of Siegler et al (2010), the bounce error after perturbations was shown to be corrected most of the time over less than 4 cycles, which is a very short time window for humans to update their internal representation of the gravity acceleration based on the visual perception of the perturbed ball acceleration. In addition, the human vision system was shown to perform very poorly at estimating object acceleration (Benguigui et al 2003; Lee et al 1997; Ripoll and Latiri 1997).

Emergent rather than explicit internal (or event-based) timing This analysis evidenced the self-organization emerging from interaction between participants and the environment when considering continuous coupling instead of intermittent parametric adaptation. The visual entrainment of the paddle movement by the ball movement is an ecological control strategy that provides crucial advantages to the embodied human motor control of action over a computational one (Schaal 2006; Beer 1998). As the CPG is continuously coupled with the ball, the timing synchronization between the agent and the environment precludes the need for an internal time-keeper. The behavior of the embodied and situated participant arises from the ongoing interaction between his/her NMS and the environment. According to the results presented in this study, the human ball-bouncing model does not need to integrate explicit internal representation of time interval. The timing emerges from the coupled system's intrinsic dynamics.

The ecology of the cycle-to-cycle correction of bounce error All the models tested in this study, as well as in Avrin et al (2016, 2017a,b); Ronsse and Sternad (2010) considered that an intermittent coupling was responsible for the paddle amplitude adaptation. This coupling takes the form of an autoregressive adaptation law relying on event-based information (the perception of the bounce error). As a consequence, it cannot be the result of some sort of continuous coupling supporting the prospective hypothesis. However, in the CPGbased models presented in this paper and in Avrin et al (2016, $2017 \mathrm{a}, \mathrm{b})$, the movement variable $(\Delta u)$ is directly linked to an 
information variable $\varepsilon$ perceived in the environment. Thus, this adaptation law stays in agreement with the ecological approach to the perception-action cycle (Warren 1988, 2006). Indeed, there is no need for high-level information processing such as the computation of an intermediate variable or internal representation. In addition, the information variable does not specify a reference state to be reached in the future, contrary to the optimal controllers presented in Ronsse et al (2010); Ronsse and Sternad (2010), which are closer to the predictive control approach. As such, these optimal controllers would suggest that the amplitude adaptation corresponds to a high-level cognitive process, as assumed by the informationprocessing and classical cognitivism approaches (Collins et al 1998). On the contrary, the adaptation law implemented in this paper directly modifies the CPG excitability $u$ when the bounce error $\varepsilon$ is perceived. With this in mind, even the auto-regressive adaptation law presented in this paper can result from a direct perception process and thus be viewed as ecological.

Benefits for robotics applications A traditional approach to robot control is to actively regulate all the relevant variables of the movement at the price of high computational costs and complex priorities management. A dissatisfaction with the performance of this traditional vision of behavioral intelligence led roboticists to consider alternative control strategies. The fields of reactive robot control (as the behavioral-based robotic approach) and emergent robot control are directly driven by the intent to take full advantage of the environment dynamics (Roennau et al 2014; Manoonpong et al 2008). The passive walker, as proposed in McGeer et al (1990), is an example of a robot using the passive stability of the locomotion task to reduce the computational cost of behavior control. An active control can complement the passive control strategy to enlarge the attraction domain of the agent-environment dynamic system and increase the behavior robustness (Collins et al 2005). Similarly, instead of overdriving the limbs, it is known that humans tune into the resonant frequency of their limbs when possible, to gain stability and save energy. Based on this observation, Williamson (1998) proposed to control rhythmic arm movements of humanoid robots by tuning into the resonant frequency of the mechanical arm using proprioceptive sensory feedback. Resonance tuning and entrainment phenomena emerging from continuous visual couplings between the agent and the environment are however much less exploited in robotics. This study demonstrated that the continuous sensorimotor feedback driving the CPG should not be limited to proprioceptive information. Robot control could greatly benefit from resonance phenomena resulting from continuous visual feedback from the environment, which allowed the paddle to be in the right place at the right time to stabilize the bouncing ball without needing any explicit internal representation of the environment or additional con- trol of the paddle acceleration at impact. The fact of being coupled with the ball velocity instead of ball position leads to a control architecture that has both a prospective advantage (velocity provides information about the future state of the ball) and a resonant tuning advantage that brings stability and energy saving.

\section{Conclusion}

The continuously changing dynamic properties of the environment require a constant adaptation of humans' movements based on task goals. Such adjustments rely upon informationmovement couplings involved during the on-going movement execution. This study investigated these couplings by analyzing the human ball-bouncing task, which presents both timing synchronization and spatial error correction processes and thus constitutes a relevant benchmark for the study of human motor control of rhythmic tasks. Previous studies already evidenced that human participants tend to hit the ball in what has been called the passive stability regime. This study brought decisive elements to explain the convergence toward this preferential limit cycle by analyzing the influence of gravity on the participants' steady-state behavior. The intervention of a resonance tuning phenomenon, resulting from the continuous coupling between the perception of the ball trajectory and the paddle trajectory, was shown to be an essential block of the human behavior model, in agreement with the strong on-line hypothesis stating that the visual control of action is achieved on-line, without requiring the use of model-based control strategies.

\section{References}

Ankarali MM, Tutkun Şen H, De A, Okamura AM, Cowan NJ (2014) Haptic feedback enhances rhythmic motor control by reducing variability, not improving convergence rate. J Neurophysiol 111(6):12861299

Arsenio AM (2000) Neural oscillator networks for rhythmic control of animats. From Animals to Animats 6:105-114

Avrin G, Makarov M, Rodriguez-Ayerbe P, Siegler IA (2016) Particle swarm optimization of Matsuoka's oscillator parameters in humanlike control of rhythmic movements. Proc IEEE American Control Conf pp 342-347

Avrin G, Makarov M, Rodriguez-Ayerbe P, Siegler IA (2017a) Dynamic stability of repeated agent-environment interactions during the hybrid ball-bouncing task. Proc Int Conf Informatics in Control, Automation and Robotics pp 486-496

Avrin G, Siegler IA, Makarov M, Rodriguez-Ayerbe P (2017b) Model of rhythmic ball bouncing using a visually controlled neural oscillator. Journal of Neurophysiology 118(4):2470-2482

Bazile C, Siegler IA, Benguigui N (2013) Major changes in a rhythmic ball-bouncing task occur at age 7 years. PLoS ONE 8(10):e74,127

Bazile C, Benguigui N, Siegler IA (2016) Development of informationmovement couplings in a rhythmical ball-bouncing task: from spaceto time-related information. Exp Brain Res 234(1):173-183 
Beer RD (1998) Framing the debate between computational and dynamical approaches to cognitive science. Behavioral and Brain Sciences 21(05):630-630

Benguigui N, Ripoll H, Broderick MP (2003) Time-to-contact estimation of accelerated stimuli is based on first-order information. Journal of Experimental Psychology: Human Perception and Performance 29(6): 1083

Bertenthal BI, Rose JL, Bai DL (1997) Perception-action coupling in the development of visual control of posture. Journal of Experimental Psychology Human Perception and Performance 23:1631-1643

Bootsma RJ, Van Wieringen PC (1990) Timing an attacking forehand drive in table tennis. Journal of experimental psychology: Human perception and performance 16(1):21

Buekers MJ, Bogaerts HP, Swinnen SP, Helsen WF (2000) The synchronization of human arm movements to external events. Neuroscience Letters 290(3): 181-184

Collins S, Ruina A, Tedrake R, Wisse M (1998) Complex-systems perspective on the "computation vs. dynamics" debate in cognitive science. Proc Conference of the Cognitive Science Society pp 710-715

Collins S, Ruina A, Tedrake R, Wisse M (2005) Efficient bipedal robots based on passive-dynamic walkers. Science 307(5712):1082-1085

Dessing JC, Peper CLE, Bullock D, Beek PJ (2005) How position, velocity, and temporal information combine in the prospective control of catching: data and model. Journal of cognitive neuroscience 17(4):668-686

Dietz V, Duysens J (2000) Significance of load receptor input during locomotion: a review. Gait \& posture 11(2):102-110

Dijkstra T, Schöner G, Gielen C (1994a) Temporal stability of the action-perception cycle for postural control in a moving visual environment. Experimental Brain Research 97(3):477-486

Dijkstra T, Schöner G, Giese MA, Gielen C (1994b) Frequency dependence of the action-perception cycle for postural control in a moving visual environment: relative phase dynamics. Biological cybernetics 71(6):489-501

Gan L, Huang Y, Zhou L, Qian C, Wu X (2015) Synchronization to a bouncing ball with a realistic motion trajectory. Scientific reports $5: 11,974$

Gibson J (1979) The ecological approach to visual perception

Goldfield EC, Kay BA, Warren WH (1993) Infant bouncing: The assembly and tuning of action systems. Child development 64(4):11281142

Grillner S (2006) Biological pattern generation: The cellular and computational logic of networks in motion. Neuron 52(5):751 - 766

Harris-Warrick RM (2011) Neuromodulation and flexibility in central pattern generator networks. Curr Opin Neurobiol 21(5):685 - 692, networks, circuits and computation

Hatsopoulos NG, Warren Jr WH (1996) Resonance tuning in rhythmic arm movements. Journal of motor behavior 28(1):3-14

Hove MJ, Iversen JR, Zhang A, Repp BH (2013) Synchronization with competing visual and auditory rhythms: bouncing ball meets metronome. Psychological Research 77(4):388-398

Iversen JR, Patel AD, Nicodemus B, Emmorey K (2015) Synchronization to auditory and visual rhythms in hearing and deaf individuals. Cognition 134:232-244

Katsumata H, Russell DM (2012) Prospective versus predictive control in timing of hitting a falling ball. Experimental brain research 216(4):499-514

Lee D, Young D, Reddish P, Lough S, Clayton T (1983) Visual timing in hitting an accelerating ball. The Quarterly Journal of Experimental Psychology 35(2):333-346

Lee D, Port NL, Georgopoulos AP (1997) Manual interception of moving targets ii. on-line control of overlapping submovements. Experimental Brain Research 116(3):421-433

Lopresti-Goodman SM, Richardson MJ, Silva PL, Schmidt R (2008) Period basin of entrainment for unintentional visual coordination.
Journal of Motor Behavior 40(1):3-10

Manoonpong P, Pasemann F, Wörgötter F (2008) Sensor-driven neural control for omnidirectional locomotion and versatile reactive behaviors of walking machines. Robotics and Autonomous Systems 56(3):265-288

Marchal-Crespo L, Bannwart M, Riener R, Vallery H (2015) The effect of haptic guidance on learning a hybrid rhythmic-discrete motor task. IEEE Trans Haptics 8(2):222-234

Matsuoka K (1985) Sustained oscillations generated by mutually inhibiting neurons with adaptation. Biol Cybern 52(6):367-376

Matsuoka K (2011) Analysis of a neural oscillator. Biol Cybern 104(45):297-304

Matsuoka K (2013) Frequency responses of a neural oscillator. [Blog post]. Retrieved from https://matsuoka1. jimdo.com/, accessed: $2017-03-06$

McGeer T, et al (1990) Passive dynamic walking. I J Robotic Res $9(2): 62-82$

Montagne G, Laurent M, Durey A, Bootsma R (1999) Movement reversals in ball catching. Experimental Brain Research 129(1):8792

Morice A, Siegler IA, Bardy B, Warren W (2007) Action-perception patterns in virtual ball bouncing: combating system latency and tracking functional validity. Exp Brain Res 181:249-265

Oullier O, De Guzman GC, Jantzen KJ, Lagarde J, Scott Kelso J (2008) Social coordination dynamics: Measuring human bonding. Social neuroscience 3(2):178-192

Pearson KG (2004) Generating the walking gait: role of sensory feedback. Progress in brain research 143:123-129

Pelah A, Barbur J, Thurrell A, Hock HS (2015) The coupling of vision with locomotion in cortical blindness. Vision research 110:286-294

Rabinovich MI, Varona P, Selverston AI, Abarbanel HD (2006) Dynamical principles in neuroscience. Reviews of modern physics 78(4): 1213

Repp BH (2005) Sensorimotor synchronization: a review of the tapping literature. Psychonomic bulletin \& review 12(6):969-992

Ripoll H, Latiri I (1997) Effect of expertise on coincident-timing accuracy in a fast ball game. Journal of Sports Sciences 15(6):573580

Roennau A, Heppner G, Nowicki M, Zöllner JM, Dillmann R (2014) Reactive posture behaviors for stable legged locomotion over steep inclines and large obstacles. In: Proc. IEEE Int. Conf. Intelligent Robots and Systems, IEEE, pp 4888-4894

Ronsse R, Sternad D (2010) Bouncing between model and data: stability, passivity, and optimality in hybrid dynamics. J Mot Behav 42(6):389-399

Ronsse R, Wei K, Sternad D (2010) Optimal control of a hybrid rhythmic-discrete task: The bouncing ball revisited. J Neurophysiol 103(5):2482-2493

Rossignol S, Dubuc R, Gossard JP (2006) Dynamic sensorimotor interactions in locomotion. Physiol Rev 86(1):89-154

de Rugy A, Wei K, Müller H, Sternad D (2003) Actively tracking 'passive' stability in a ball bouncing task. Brain Res 982(1):64 - 78

Russell DM, Sternad D (2001) Sinusoidal visuomotor tracking: intermittent servo-control or coupled oscillations? Journal of Motor Behavior 33(4):329-349

Schaal S (2006) Dynamic movement primitives-a framework for motor control in humans and humanoid robotics. In: Adaptive motion of animals and machines, Springer, pp 261-280

Schaal S, Sternad D, Atkeson CG (1996) One-handed juggling: A dynamical approach to a rhythmic movement task. J Mot Behav 28(2):165-183

Schmidt R, Turvey MT (1994) Phase-entrainment dynamics of visually coupled rhythmic movements. Biological cybernetics 70(4):369376

Schmidt R, Bienvenu M, Fitzpatrick P, Amazeen P (1998) A comparison of intra-and interpersonal interlimb coordination: Coordination 
breakdowns and coupling strength. Journal of Experimental Psychology: Human Perception and Performance 24(3):884

Schmidt R, Richardson MJ, Arsenault C, Galantucci B (2007) Visual tracking and entrainment to an environmental rhythm. Journal of Experimental Psychology: Human Perception and Performance 33(4):860

Schmidt RC, Carello C, Turvey MT (1990) Phase transitions and critical fluctuations in the visual coordination of rhythmic movements between people. Journal of experimental psychology: human perception and performance 16(2):227

Siegler IA, Bardy BG, Warren WH (2010) Passive vs. active control of rhythmic ball bouncing: the role of visual information. J Exp Psychol Hum Percept Perform 36(3):729-50

Siegler IA, Bazile C, Warren W (2013) Mixed control for perception and action: timing and error correction in rhythmic ball-bouncing. Exp Brain Res 226(4):603-615

Sternad D, Duarte M, Katsumata H, Schaal S (2001) Bouncing a ball: tuning into dynamic stability. J Exp Psychol Hum Percept Perform 27(5):1163

Sylos-Labini F, Ivanenko YP, Cappellini G, Portone A, MacLellan MJ, Lacquaniti F (2013) Changes of gait kinematics in different simulators of reduced gravity. Journal of motor behavior 45(6):495505

Taga G (1995) A model of the neuro-musculo-skeletal system for human locomotion. Biol Cybern 73(2):97-111

Torre K, Balasubramaniam R (2009) Two different processes for sensorimotor synchronization in continuous and discontinuous rhythmic movements. Experimental Brain Research 199(2):157-166

Tufillaro N, Mello T, Choi Y, Albano A (1986) Period doubling boundaries of a bouncing ball. Journal de Physique 47(9):1477-1482

Van Der Steen MC, Keller PE (2013) The adaptation and anticipation model (adam) of sensorimotor synchronization. Frontiers in human neuroscience 7:253

Varlet M, Coey CA, Schmidt R, Richardson MJ (2012) Influence of stimulus amplitude on unintended visuomotor entrainment. Human movement science 31(3):541-552

Varlet M, Coey CA, Schmidt R, Marin L, Bardy BG, Richardson MJ (2014) Influence of stimulus velocity profile on rhythmic visuomotor coordination. Journal of Experimental Psychology: Human Perception and Performance 40(5):1849

Warren WH (1988) Action modes and laws of control for the visual guidance of action. Advances in psychology 50:339-379

Warren WH (2006) The dynamics of perception and action. Psychol Rev 113:358-389

Washburn A, Coey CA, Romero V, Richardson MJ (2014) Visual multifrequency entrainment: can 1:2, 2: 3, and 3: 4 coordination occur spontaneously? Journal of motor behavior 46(4):247-257

Wei K, Dijkstra T, Sternad D (2007) Passive stabiliy and variability: indicators for passive stability and active control in a rhythmic task. J Neurophysiol 98:2633-2646

Wei K, Dijkstra TMH, Sternad D (2008) Stability and variability: Indicators for passive stability and active control in a rhythmic task. J Neurophysiol 99(6):3027-3041

White O, Bleyenheuft Y, Ronsse R, Smith AM, Thonnard JL, Lefevre $\mathrm{P}$ (2008) Altered gravity highlights central pattern generator mechanisms. Journal of neurophysiology 100(5):2819-2824

Williamson M (1998) Neural control of rhythmic arm movements. Neural Networks 11:1379-1394

Williamson M (1999) Designing rhythmic motions using neural oscillators. Proc IEEE/RSJ Int Conf on Intelligent Robots and Systems (IROS) 1:494-500

Wimmers RH, Beek PJ, van Wieringen PC (1992) Phase transitions in rhythmic tracking movements: A case of unilateral coupling. Human Movement Science 11(1):217-226

Wolpert DM, Ghahramani Z (2000) Computational principles of movement neuroscience. nature neuroscience 3:1212-1217
Yagoubi M, Sandou G (2011) Particle Swarm Optimization for the design of Ho static output feedbacks. Proc IFAC World Congr

Zhang D, Zhu X, Poignet P (2009) Coupling of central and peripheral mechanism on tremor. Proc IEEE/EMBS Int Conf Neural Engineering pp 649-652

Zhao H, Warren WH (2015) On-line and model-based approaches to the visual control of action. Vision research 110:190-202 